\title{
Desarrollo de un modelo de gasificación en equilibrio químico para evaluar el potencial energético del cuesco en plantas extractoras de aceite de palma en Colombia
}

\section{Development of a Chemical Equilibrium Gasification Model to Evaluate the Energy Potential of the Palm Kernel Shells from Palm Oil Extraction Industries in Colombia}

\author{
DOI: http://doi.org/10.17981/ingecuc.14.2.2018.06 \\ Artículo de investigación científica. Fecha de recepción:07/04/2018. Fecha de aceptación:06/07/2018 \\ Daniel Andrés Quintero Coronel \\ Universidad del Norte. Barranquilla (Colombia) \\ Universidad Francisco de Paula Santander. Ocaña (Colombia) \\ quinteroda@uninorte.edu.co \\ Yuhan Lenis Rodas \\ Universidad del Norte. Barranquilla (Colombia) \\ ylenis@uninorte.edu.co \\ Lesme Antonio Corredor Martínez \\ Universidad del Norte. Barranquilla (Colombia) \\ lcorredo@uninorte.edu.co
}

Para citar este artículo:

D. Quintero Coronel, Y. Lenis Rodas y L. Corredor Martínez, "Desarrollo de un modelo de gasificación en equilibrio químico para evaluar el potencial energético del cuesco en plantas extractoras de aceite de palma en Colombia,” INGE CUC, vol. 14, no. 2, pp. 62-70, 2018. DOI: http://doi.org/10.17981/ingecuc.14.2.2018.06

\section{Resumen}

Introducción- En las industrias de extracción de aceite de palma, por cada 10 ton de racimos de fruto fresco (RFF) procesados, se producen cerca de $3700 \mathrm{~kg}$ de residuos con Poder Calorífico Inferior (PCI) de alrededor de $18 \mathrm{MJ} / \mathrm{kg}$. Estos residuos, compuestos principalmente por racimos de frutos vacíos, fibra y cuesco podrían ser utilizados para generación de electricidad o vapor supliendo de manera parcial o total la demanda de energía de las empresas del sector. De estos residuos, el que mejor se adapta para generación de electricidad a partir de biomasa, en rangos menores a $2 \mathrm{MW}$, es el cuesco, el cual puede ser utilizado en sistemas de gasificación de lecho fijo acoplados a motor generador.

Objetivo- Evaluar el potencial energético del cuesco de palma de aceite para la generación de electricidad utilizando gasificación en lecho fijo acoplada a motor generador.

Metodología-Se desarrolló un modelo de gasificación en equilibrio químico que permite estimar la composición de gases y, por tanto, el potencial energético de los residuos de cuesco de palma. El modelo permite analizar variaciones en el proceso debidas a cambios en agente gasificante (AG), composición y contenido de humedad de la biomasa. Los resultados son validados utilizando información reportada en la literatura. El modelo es utilizado para analizar el potencial energético de los residuos de una planta extractora típica de 10000 ton $\mathrm{RFF} / \mathrm{mes}$.

Conclusiones- Se estima que por cada $22 \mathrm{~kg} / \mathrm{h}$ de cuesco se producen aproximadamente $70 \mathrm{~kg} / \mathrm{h}$ de gas con composición promedio de $12,5 \% \mathrm{H}_{2}, 21,8 \% \mathrm{CO}, 9,5 \% \mathrm{CO}_{2}, 56 \% \mathrm{~N}_{2}$ y trazas de $\mathrm{CH}_{4}$ con poder calorífico inferior (PCI) cercano a 4,1 $\mathrm{MJ} /$ $\mathrm{Nm}_{3}$. Lo anterior, cuando el proceso opera con una relación de equivalencia (ER) de 0,33 y humedad de biomasa de $15 \%$ w.t. Así, utilizando el gas como combustible para un conjunto motorgenerador, la demanda eléctrica de una planta extractora de aceite puede ser suplida en su totalidad, empleando menos del $85 \%$ del cuesco resultante del proceso.

Palabras clave- Cuesco de palma; gasificación en lecho fijo; aspen plus; equilibrio químico; potencial energético

\begin{abstract}
Introduction- In palm oil extraction plants, for every 10 tons of fresh fruit bunches (FFB) that are processed, an estimate of $3700 \mathrm{~kg}$ of waste are produced. This waste, consisting of empty fruit bunches, fibers, and kernels, mainly, has a lower heating value (LHV) of about $18 \mathrm{MJ} / \mathrm{kg}$. This waste can be considered a by-product as it is possible to be used for steam generation or electricity production to completely or partially cover the energy demand of oil palm processing plants. Among these, kernels are the best option for biomass power generation in fixed-bed gasifiers coupled to motor or generator sets for power below $2 \mathrm{MW}$
\end{abstract}

Objective- Evaluate energy potential of oil palm kernel for power generation in typical oil palm extraction plants trough fixed bed gasification coupled to motor/generator sets.

Methodology-A chemical equilibrium model was developed to estimate gas composition and, therefore, energy potential of palm kernel biomass from extractive industries. This tool enables analyzing process variations caused by changes in the gasifying agent, composition and moisture content of biomass. The model was used to analyze kernel energy potential from a typical plant that processes 10000 ton of fresh fruit bunches per month. Model results were validated using data from literature. The model is used to analyze the energy potential of waste from a typical 10000 ton RFF/month extractor plant.

Conclusions- It is estimated that for every $22 \mathrm{~kg} / \mathrm{h}$ of oil palm kernel, approximately $70 \mathrm{~kg} / \mathrm{h}$ of gas are produced with an average composition of $12.5 \% \mathrm{H}_{2}, 21.8 \% \mathrm{CO}, 9.5 \% \mathrm{CO}_{2}$, $56 \% \mathrm{~N}_{2}$ and traces of $\mathrm{CH}_{4}$, with a low heat value (LHV) close to $4.1 \mathrm{MJ} / \mathrm{Nm}_{3}$. According to the results, the total electricity demand of a typical plant can be supplied using a gasificationbased system feed with $\sim 85 \%$ of the kernel from the extraction process.

Keywords- Palm kernel shell, fixed bed gasification; aspen plus; chemical equilibrium; energy potential. 


\section{INTRODUCCIÓN}

El crecimiento acelerado de la población mundial ha derivado en el incremento de la demanda de combustibles fósiles, los cuales, además de ser finitos, causan daños irreversibles al medio ambiente [1]. Esta problemática pone en evidencia la necesidad de diversificar la canasta energética global con recursos renovables de bajo impacto ambiental. Entre las fuentes alternativas de mayor potencial se encuentra la biomasa, la cual puede ser transformada en combustibles líquidos, sólidos o gaseosos de fácil aprovechamiento en las máquinas térmicas convencionales [2]. Los beneficios ambientales asociados al uso de este recurso se maximizan cuando se considera biomasa residual. En Colombia, el sector de extracción de aceite de palma es uno de los más importantes generadores de biomasa por hectárea cultivada en comparación con otros cultivos oleaginosos y bioenergéticos. Por cada 10000 kg de racimos de fruto fresco (RFF) procesados, se producen cerca de $2000 \mathrm{~kg}$ de racimos de frutos vacíos, $1300 \mathrm{~kg}$ de fibra y $600 \mathrm{~kg}$ de cuesco, esto es, alrededor de 4000 $\mathrm{kg}$ de residuos, cuyas densidades energéticas promedio oscilan alrededor de $18 \mathrm{MJ} / \mathrm{kg}$ [3], [4]. El potencial energético de estos residuos puede ser aprovechado para la generación de fluidos energéticos que suplan, parcial o totalmente, la demanda energética de las extractoras. Considerando que la energía primaria de la fibra es aproximadamente 3 veces la energía requerida para la generación de vapor de baja presión (entre 3 y 4 bar) requerido en los sub-procesos de esterilización y digestión, en este trabajo se analiza la generación de electricidad a partir de otro de los residuos de alto potencial: el cuesco, cuya densidad aparente, tamaño de partícula y poder calorífico ( 19 $\mathrm{MJ} / \mathrm{kg}$ ), ponen en evidencia su alto potencial para sistemas de gasificación de lecho fijo.

Para una planta que procese alrededor de 10000 ton RFF/mes, el uso del cuesco en un sistema de generación con eficiencias globales alrededor del $20 \%$ permitiría generar alrededor de $1 \mathrm{MWe}$, rango de potencias en los que la tecnología de gasificación en lecho fijo equicorriente acoplado a motor generador es la que mejor se adapta para la transformación energética [5]-[7]. Este sistema es considerado uno de los de mayor madurez tecnológica para generación eléctrica en potencias inferiores a $2 \mathrm{MWe}$. El proceso se caracteriza por la generación de un gas con poder calorífico de entre 4-6 $\mathrm{MJ} / \mathrm{Nm}^{3}$ [8], el cual es considerado bajo, en comparación con gas natural (aprox. $39 \mathrm{MJ} / \mathrm{Nm}^{3}$ ), pero que puede ser utilizado en un conjunto de motor de combustión interna-generador para la generación de electricidad. Pese a que para este proceso se estiman eficiencias combinadas gasificador-motor-generador de entre 16-24\%, buscando mayor confiabilidad de los resultados, en el presente estudio se desarrolla un modelo en equilibrio termoquímico que permite, además de analizar el potencial energético, evaluar el efecto que tendrían factores como el aumento de la humedad y la disminución del poder calorífico de la biomasa sobre la generación de electricidad.

En el ámbito de gasificación se han desarrollado modelos que permiten verificar el comportamiento de materias primas para diferentes reactores. En este sentido, Paviet et al. [9] desarrollaron un modelo de gasificación de biomasa en equilibrio termoquímico para un reactor de corriente descendente utilizando el software Aspen Plus. El modelo fue validado usando datos de un gasificador experimental de corriente descendente. Las simulaciones permitieron predecir la composición del gas, tanto en la zona de pirolisis como a la salida del reactor. Dicho modelo fue adaptado a las condiciones operacionales de un gasificador ubicado en la Technical University of Denmark (DTU). En dicho trabajo, los autores asumen que el proceso alcanza el estado de equilibrio en condiciones adiabáticas o isotérmicas y que los productos están compuestos principalmente de $\mathrm{H}_{2}, \mathrm{CO}, \mathrm{CO}_{2}, \mathrm{CH}_{4}$, y $\mathrm{H}_{2} \mathrm{O}$, ignorando la presencia de alquitranes. Trabajos similares para modelado del proceso de gasificación en lecho fijo equicorriente usando Aspen Plus han sido desarrollado por diversos autores [10]-[14], éstos tienen en común los bloques de trabajo del programa: Ryield, encargado de la descomposición de la materia prima utilizada, y RGibbs, encargado de simular la oxidación del proceso. Los modelos predicen adecuadamente la composición del gas obtenido asumiendo que el proceso alcanza el equilibrio químico mediante la minimización de la energía libre de Gibbs, por otro lado, se diferencian en los tipos de agente gasificante utilizados: aire, vapor de agua y mezclas. Alembath [15] desarrolló un modelo cinético de la gasificación en un reactor de corriente descendente. Este autor simuló las zonas de secado, pirolisis, combustión y gasificación. Para las zonas de secado y pirolisis, el autor utilizó un reactor Ryield y un Rcstr (especificando las reacciones que ocurren en cada zona), dos reactores Rcstr fueron usados para simular las zonas de combustión y gasificación, respectivamente. En este tipo de reactores se especifican las reacciones que ocurren en cada etapa. El autor varía la relación aire/combustible y la temperatura de gasificación para ver su influencia sobre la composición del gas obtenido, validando los resultados mediante comparación directa con lo publicado en [16]-[18]. La determinación de la concentración éste en la gasificación mediante modelos de equilibrio químico resulta en una herramienta de gran utilidad para la evaluación del potencial energético de cualquier residuo, puesto que permite obtener tanto cantidad como poder calorífico del gas.

A pesar de la importancia del desarrollo de estudios como el presente para fomentar el aprovechamiento de recursos renovables, no se encuentra en la literatura ningún estudio que busque determinar el potencial del cuesco de palma para la generación de electricidad en una planta extractora de aceite, partiendo de resultados detallados obtenidos mediante modelos del proceso. 


\section{Metodología}

De acuerdo con la Federación Nacional de Cultivadores de Palma de Aceite de Colombia (FEDEPALMA) y Briceño et al. [19], las plantas extractoras de aceite de palma tienen el potencial de generar energía eléctrica mediante la utilización de los residuos del proceso a través de tecnologías de conversión energética. La energía generada puede destinarse para autoconsumo o autoconsumo y venta de excedentes.

En FEDEPALMA y Briceño et al. [19], se estimó que por cada $10000 \mathrm{~kg}$ de racimos de fruto fresco (RFF) procesados se producen cerca de $2000 \mathrm{~kg}$ de tusa, $1300 \mathrm{~kg}$ de fibra y $600 \mathrm{~kg}$ de cuesco, esto es, alrededor de $4000 \mathrm{~kg}$ de residuos (Fig. 1) que pueden ser aprovechados en procesos de generación de energía térmica o eléctrica en estas industrias.

Considerando la producción de residuos para una extractora que procese 10000 ton RFF/mes (caso base de estudio), la potencia a generar a partir de biomasa en sistemas con eficiencias globales cercanas a $20 \%(\sim 1,2 \mathrm{~kg} / \mathrm{kWeh})$ estaría alrededor de 1 MWe. Para este nivel de potencia, la tecnología que mejor se adapta para la conversión energética es la gasificación en lecho fijo equicorriente acoplada a motor generador [5]. Teniendo en cuenta este resultado preliminar, se desarrolla un modelo en equilibrio químico que permite estimar con un mayor nivel de detalle la potencia a generar partiendo del gas producido en el proceso y de sus variaciones, debidas a cambios en parámetros operacionales, como lo son la humedad de la biomasa y la relación de equivalencia. También son consideradas variaciones en la cantidad de RFF procesados mes a mes, la cual puede depender tanto de factores climáticos como socioeconómicos. El perfil de variación se deriva de la información de producción nacional de aceite reportada por FEDEPALMA para el año 2015 [20], pues no se cuenta con estudios más recientes.

Para el modelo del proceso se considera el aire como agente oxidante debido a su disponibilidad. Aunque podrían ser empleados oxígeno puro y vapor de agua, estos no son considerados debido a que su uso implica el uso de equipos adicionales, aumentando el costo y el nivel de complejidad del sistema.

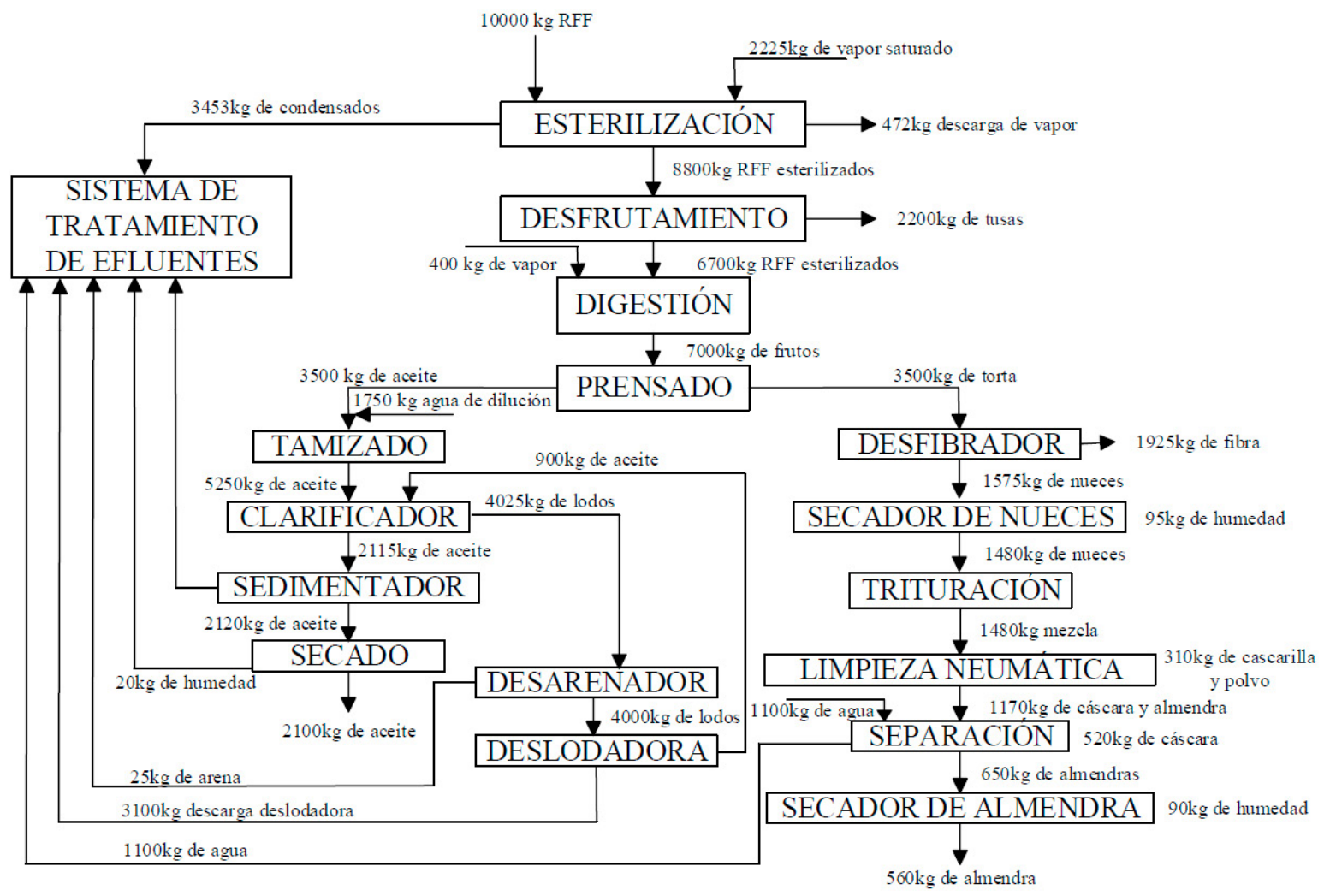

Fig. 1. Diagrama del proceso para una planta extractora que procesa $10.000 \mathrm{~kg}$ de RFF. Fuente: adaptado de [21]. 


\section{A. Desarrollo del modelo}

El esquema del modelo desarrollado en este trabajo se presenta en la Fig. 2. Este cuenta con reactores específicos para simular las etapas de secado, pirolisis, oxidación y reducción características de este tipo de procesos.

Aspen Plus ${ }^{\circledR}$ posee, dentro de sus librerías, lo que se conoce como compuestos convencionales y no convencionales, es decir, compuestos que el software puede reconocer o no. Dado que las materias primas a utilizar se encuentran dentro de la categoría de 'no convencionales', es necesario suministrar al software los análisis próximo y último de la biomasa. Así mismo, debe proporcionarse el método de trabajo que el software utilizará para estimar las propiedades del proceso. En este caso, se ha utilizado el método de la ecuación de estado de Peng-Robinson con la función alpha de Boston-Matias (PR-BM) para calcular todas las propiedades físicas de los compuestos convencionales, mientras que la estimación de la entalpía y densidad de los compuestos no convencionales ha sido calculada por los métodos HCOALGEN y DCOALIGT, de acuerdo con lo publicado por [21].

El modelo se desarrolla para gasificación con aire, bajo la suposición de que el proceso alcanza equilibrio químico y que las especies formadas son $\mathrm{CO}, \mathrm{CO}_{2}, \mathrm{H}_{2}, \mathrm{CH}_{4}, \mathrm{~N}_{2}, \mathrm{H}_{2} \mathrm{O}$, compuestos principales en el caso de utilizar aire como agente oxidante.

Buscando validar los resultados a partir de información disponible en la literatura, se consideran los resultados reportados por Jayah et al. [16], quien utilizó madera proveniente del árbol de caucho (RW, por sus siglas en inglés) como materia prima y la gasificó en un reactor de lecho fijo equicorriente bajo condiciones atmosféricas. El análisis elemental y próximo de este material, así como del cuesco de palma utilizado como referencia, puede visualizarse en la tabla 1.

Tabla 1. ANÁlisis PRÓXimo y Último PARA la Biomasa RW, CONSIDERADA PARA VALIDACIÓN.

\begin{tabular}{|c|c|c|c|c|c|}
\hline \multicolumn{2}{|c|}{$\begin{array}{c}\text { Análisis próximo } \\
\text { (\% masa) }\end{array}$} & \multicolumn{4}{c|}{$\begin{array}{c}\text { Análisis último } \\
\text { (\% masa) }\end{array}$} \\
\hline & $\mathrm{RW}$ & Cuesco & & $\mathrm{RW}$ & Cuesco \\
\hline $\begin{array}{c}\text { Carbono fijo } \\
\text { (FC) }\end{array}$ & 19,2 & 13,71 & $\mathrm{C}$ & 50,6 & 48,75 \\
\hline $\begin{array}{c}\text { Materia } \\
\text { volátil (VM) }\end{array}$ & 80,1 & 76,82 & $\mathrm{H}$ & 6,5 & 5,55 \\
\hline $\begin{array}{c}\text { Humedad } \\
\text { (MC) }\end{array}$ & $0-30$ & 5,91 & $\mathrm{O}$ & 42 & 35,33 \\
\hline Cenizas & 0,7 & 3,56 & $\mathrm{~N}$ & 0,2 & 0,80 \\
\hline & & & $\mathrm{S}$ & 0,0 & 0,10 \\
\hline
\end{tabular}

Fuente: autores y tomado de Jayah et al. [16].

\section{B. Etapa de secado}

El secado de la materia prima depende de su humedad, la cual debería estar por debajo del $25 \%$ en el caso de la gasificación para que el proceso conserve su carácter autotérmico. El bloque utilizado en esta etapa es un Rstoic (SECADO), el cual se encarga de calcular el secado de la materia prima (MATERIAL) con base en la fracción de conversión o de producción de alguno de los elementos que participan en la reacción, utilizando una línea declarada en FORTRAN [21], la cual actúa en la unidad calculadora $\left(\mathrm{H}_{2} \mathrm{O}\right.$ CALCULATOR, no mostrada en la Fig. 2). El bloque separador (SEPARA) se encarga de separar la materia prima que ha sido secada (MATSECO) de la cantidad de vapor de agua (VAPOR), y este flujo de vapor es considerado posteriormente como parte del agente gasificante. El subproceso de secado fue simulado de esta manera con el fin de simplificar el modelo, luego, el flujo MATSECO se lleva a la siguiente etapa del modelo.

\section{Etapa de pirolisis}

La pirolisis o desvolatilización empieza a una temperatura cercana a los $160{ }^{\circ} \mathrm{C}$ hasta los $450{ }^{\circ} \mathrm{C}$ [22]. En este proceso, los enlaces químicos entre las cadenas de aromáticos de la materia prima son 'liberados' generando fragmentos con pesos moleculares mucho más pequeños. Las partículas con peso molecular bajo son liberadas en forma de gases livianos y alquitranes, mientras que los de alto peso molecular permanecen aún en desvolatilización, hasta que son transformados en carbonizado (CHAR) [23].

Para el caso del modelo, se ha utilizado un bloque Ryield, el cual se usa cuando se desconocen las reacciones químicas que se llevan a cabo pero se conocen datos de la distribución de la formación de productos de la reacción, correlaciones, fórmulas empíricas, donde se determina la formación de cada uno de los productos [24]. Este bloque descompone los elementos no convencionales de la materia prima utilizada en sus elementos constituyentes $\mathrm{C}, \mathrm{H}$, $\mathrm{N}, \mathrm{O}, \mathrm{S}, \mathrm{H}_{2} \mathrm{O}, \mathrm{Cl}$, entre otros [21], de acuerdo con el análisis último suministrado al software por medio de un bloque calculador controlado por las declaraciones suministradas en FORTRAN.

El Ryield (DESVOL) tiene como entrada el flujo MATSECO del bloque anterior y tiene como salida el flujo PRODESVO, el cual está formado por gases livianos y CHAR, este último se ha modelado como carbón sólido de acuerdo con lo establecido por [24]. La temperatura del Ryield puede variarse desde los $150^{\circ} \mathrm{C}$ hasta los $450^{\circ} \mathrm{C}$ si desea saberse la influencia del factor temperatura sobre la línea de flujo PRODESVO. 


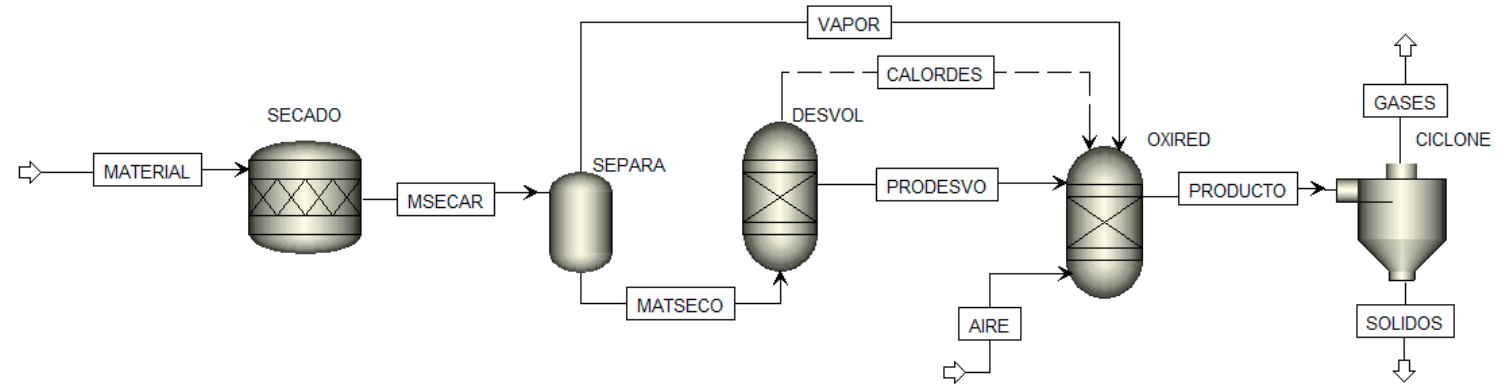

Fig. 2. Modelo desarrollado. Fuente: autores.

\section{A. Etapa de oxidación y reducción}

La oxidación y la reducción ocurre cuando los gases liberados en la pirolisis son oxigenados con un medio gasificante. Este medio puede ser oxígeno puro, aire, vapor de agua o mezclas de los anteriores. El modelo presentado aquí se ha desarrollado para aire como agente gasificante. Las reacciones que ocurren durante el proceso se presentan en las ecuaciones 1-10 [25]- [26].

Oxidación parcial:

$\mathrm{C}_{(\mathrm{S})}+0.5 \mathrm{O}_{2} \rightarrow \mathrm{CO}$

Oxidación total:

$\mathrm{C}_{(\mathrm{S})}+\mathrm{O}_{2} \rightarrow \mathrm{CO}_{2}$

Reacción de Boudouard:

$\mathrm{C}_{(S)}+\mathrm{CO}_{2(g)} \leftrightarrow 2 \mathrm{CO}_{2(g)}$

Reacción de metanización:

$\mathrm{C}_{(\mathrm{S})}+2 \mathrm{H}_{2(\mathrm{~g})} \leftrightarrow \mathrm{CH}_{4(g)}$

Generación de vapor:

$\mathrm{C}_{(\mathrm{S})}+\mathrm{H}_{2} \mathrm{O}_{(\mathrm{g})} \leftrightarrow \mathrm{CO}_{(\mathrm{g})}+\mathrm{H}_{2(\mathrm{~g})}$

Agua-Gas shift:

$\mathrm{CO}_{(g)}+\mathrm{H}_{2} \mathrm{O}_{(g)} \leftrightarrow \mathrm{CO}_{2(g)}+\mathrm{H}_{2(g)}$

Reformado de metano con vapor:

$\mathrm{CH}_{4(g)}+\mathrm{H}_{2} \mathrm{O}_{(g)} \rightarrow \mathrm{CO}_{(g)}+3 \mathrm{H}_{2(g)}$

Formación de $\mathrm{H}_{2} \mathrm{~S}$ :

$H_{2(g)}+S_{(S)} \rightarrow H_{2} S_{(g)}$

Formación de $\mathrm{NH}_{3}$ :

$0.5 \mathrm{~N}_{2}+1.5 \mathrm{H}_{2} \rightarrow \mathrm{NH}_{3(\mathrm{~g})}$

Formación de $\mathrm{HCl}$ :

$\mathrm{Cl}_{2(g)}+\mathrm{H}_{2(g)} \rightarrow 2 \mathrm{HCl}_{(g)}$
El bloque usado para simular el proceso de oxidación y reducción es un RGibbs (OXIRED), el cual se encarga de estimar la composición de los gases de salida cuando el proceso alcanza el equilibrio químico. OXIRED recibe el flujo PRODESVO proveniente del bloque DESVOL, el cual posee los compuestos convencionales en forma de gases livianos y carbono como sólido. Una vez en OXIRED, un flujo de aire a condiciones ambientales es inyectado al reactor provocando las reacciones de oxidación, así mismo, el flujo de vapor liberado en la etapa de secado es inyectado también en este reactor. La temperatura que alcanza el reactor puede ser estimada al considerar un flujo de calor proveniente del bloque DESVOL; este parámetro es importante, pues permite saber la máxima temperatura alcanzada por el reactor cuando llega al equilibrio químico.

Dado que la salida de flujo PRODUCTO es una mezcla de gases y sólidos (cenizas), se hace necesario separarlos con el fin de dividirlo en dos flujos para obtener el gas producto que sería usado en aplicaciones reales de un proceso dado. La separación se simuló mediante el bloque SSplit (CICLO$\mathrm{NE}$ ), los gases se consideran para estar formados principalmente por $\mathrm{H}_{2} \mathrm{O}, \mathrm{N}_{2}, \mathrm{O}_{2}, \mathrm{H}_{2}, \mathrm{CH}_{4}, \mathrm{CO}$ y $\mathrm{CO}_{2}$, mientras que los sólidos se consideran como cenizas.

\section{Resultados}

\section{A. Validación del modelo}

El modelo fue validado con los datos publicados por Jayah et al. [16]. En la tabla 2 se muestran

Tabla 2. Condiciones para Validación del Modelo.

\begin{tabular}{|c|c|c|c|c|c|c|c|c|c|}
\hline Prueba & $\mathbf{1}$ & $\mathbf{2}$ & $\mathbf{3}$ & $\mathbf{4}$ & $\mathbf{5}$ & $\mathbf{6}$ & $\mathbf{7}$ & $\mathbf{8}$ & $\mathbf{9}$ \\
\hline $\begin{array}{c}\text { Humedad } \\
(\%)\end{array}$ & 18,5 & 16 & 14,7 & 16 & 15,2 & 14 & 14,7 & 13,8 & 12,5 \\
\hline ER & 0,31 & 0,332 & 0,357 & 0,296 & 0,32 & 0,345 & 0,28 & 0,308 & 0,356 \\
\hline
\end{tabular}

Fuente: autores. 

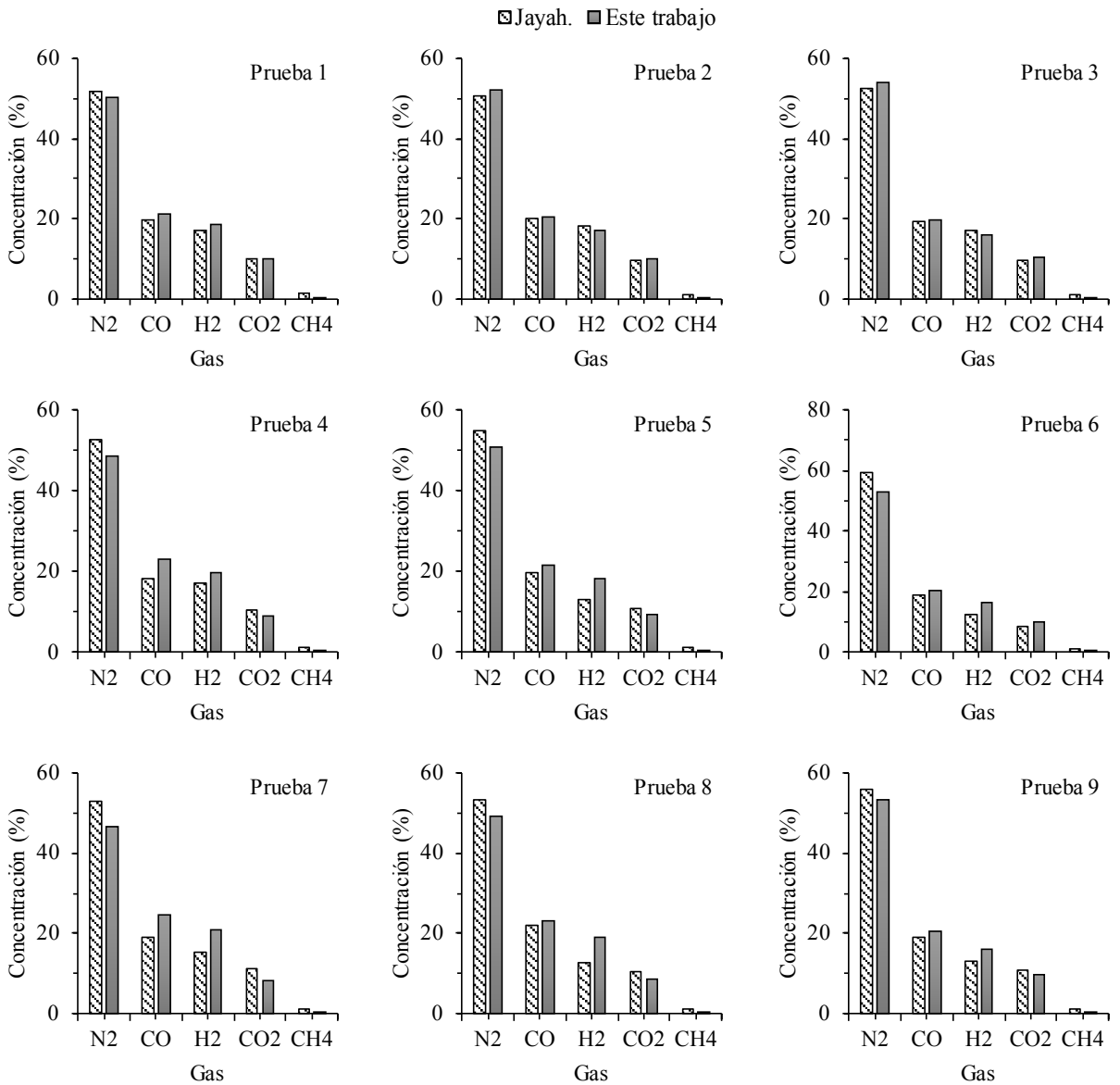

Fig. 3. Concentraciones de gas en porcentaje. Fuente: autores y adaptado de Jayah et al. [16]

las condiciones bajo las cuales se corrieron las pruebas para una temperatura de $1000{ }^{\circ} \mathrm{C}$. El flujo de aire suministrado es de $55,6 \mathrm{~kg} / \mathrm{h}$, el flujo de biomasa se varió con el fin de obtener la relación de equivalencia (ER) planteada por el autor. La Fig. 3 muestra las composiciones de gas obtenido para las condiciones mostradas en la tabla 2 .

Como puede apreciarse en la Fig. 3, el modelo predice adecuadamente las composiciones del gas para $\mathrm{CO}, \mathrm{CO}_{2}, \mathrm{~N}_{2}$, y $\mathrm{H}_{2}$, mientras que la composición de $\mathrm{CH}_{4}$ es subestimada. Este resultado coincide con lo obtenido por Paviet et al. [9] y se debe principalmente a la condición de presión ambiente del reactor y a las temperaturas alcanzadas, las cuales no favorecen las reacciones que dan lugar a la formación de este compuesto.

Por otro lado, la baja concentración de este compuesto puede ocasionar que su estimación esté en una zona de baja precisión del equipo de medición.

\section{B. Análisis de sensibilidad del modelo}

La Fig. 4 muestra el efecto de la variación de la humedad contenida en la biomasa (cuesco de palma de aceite) para una relación de equivalencia de 0,337 sobre la composición de $\mathrm{N}_{2}, \mathrm{CO}, \mathrm{CO}_{2}, \mathrm{CH}_{4}$ y $\mathrm{H}_{2}$ y vapor de agua.

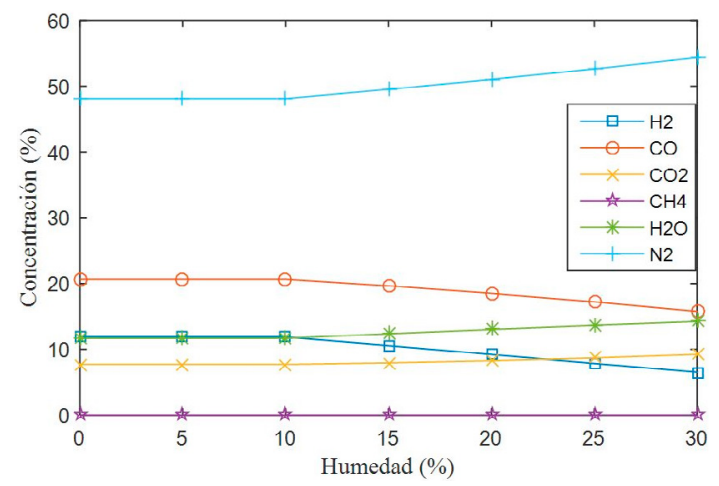

Fig. 4. Análisis de sensibilidad de concentración de gases como función de la humedad de la biomasa. Fuente: autores. 


\section{DESARROLLO DE UN MODELO DE GASIFICACIÓN EN EQUILIBRIO QUÍMICO PARA EVALUAR EL POTENCIAL ENERGÉTICO DEL CUESCO EN PLANTAS EXTRACTORAS DE ACEITE DE PALMA EN COLOMBIA}

El CO y el $\mathrm{H}_{2}$ disminuyen mientras el $\mathrm{CO}_{2}$ presenta una tendencia leve al alza, caso similar ocurre con el $\mathrm{N}_{2}$ y el $\mathrm{H}_{2} \mathrm{O}$, los cuales aumentan con el incremento de la humedad. Por otro lado, la variación del CH4 no es observable dada la baja concentración de este gas. Este comportamiento se debe a que la reacción de gasificación con vapor se ve favorecida por el aumento de la concentración de agua en los reactivos luego de la etapa de secado. Para variaciones de la humedad entre $0-10 \%$ no se observan efectos significativos sobre la composición del gas pese al aumento del gasto energético que implicaría secar la biomasa para llevar su contenido de humedad por debajo del $10 \%$. De acuerdo con experiencias previas del grupo de investigación, a nivel experimental no es posible desarrollar gasificación para biomasas con humedades superiores al $25 \%$, resultado que es corroborado mediante el modelo debido a que para estas humedades disminuye considerablemente la concentración de gases combustibles mientras aumenta la concentración de nitrógeno.

\section{Análisis de potencial energético del cuesco}

El poder calorífico inferior del gas (PCI, o LHV por sus siglas en inglés) es estimado a partir de la concentración del gas, haciendo uso de la ecuación (11) [10]. Este parámetro, junto con la tasa de generación de gas, es utilizado para la estimación del potencial energético de una determinada biomasa. La Fig. 5 muestra el PCI para los valores obtenidos al variar la humedad de 10 a $20 \%$ para relaciones de equivalencia de 0,302 hasta 0,377 .

$$
P C I_{(\text {gasproduto })}=\left(30 * n C O+25.7 * n H_{2}+85.4 * n \mathrm{CH}_{4} * 4.2\right. \text { (11) }
$$

Donde $n$ representa la fracción molar del compuesto que acompaña.

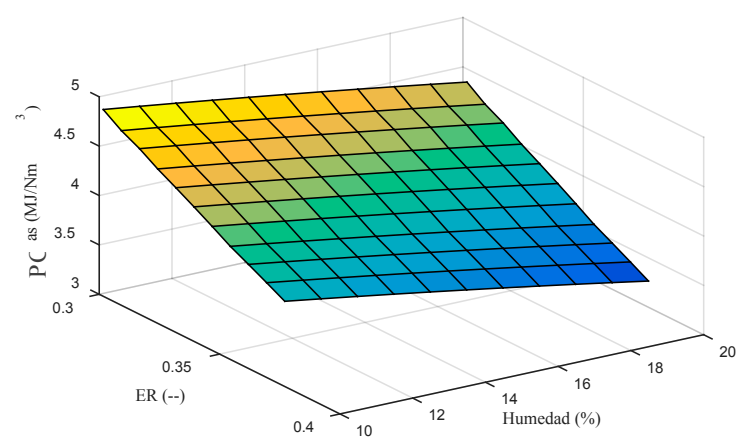

Fig. 5. Variación del PCI con el porcentaje de humedad para relaciones de equivalencia dadas. Fuente: autores.

Se puede observar que el mayor PCI ocurre para baja ER y baja humedad de la biomasa, esto se presenta debido a que el aumento de la humedad disminuye la temperatura del proceso, haciendo que éste sea muy inestable, lo que, además, reduce las composiciones de $\mathrm{CO}, \mathrm{H}_{2}$ y $\mathrm{CH}_{4}$, los cuales son los mayores contribu- yentes en la estimación del PCI, como se puede notar en la Fig. 5. Respecto a la ER, el resultado se debe a la menor disponibilidad de aire para el proceso. No se analizaron menores ER debido a que, experimentalmente, el proceso ha presentado inestabilidades cuando este parámetro baja de 0,3 .

\section{Potencial de generación}

Se toma como caso de estudio una planta de aprovechamiento de 10000 toneladas/mes. En este tipo de plantas, aproximadamente el $3 \%$ del fruto procesado corresponde a los residuos de cuesco, los cuales son los utilizados en este análisis [3]. La Fig. 6 muestra la potencia de la planta requerida para aprovechar energéticamente estos residuos. En la figura, se analiza el efecto de la humedad, variada entre 10 y $20 \%$, y las relaciones de equivalencia, entre 0,3 y 0,33 , intervalo en el cual se han obtenido desempeños experimentales satisfactorios con el uso de otras biomasas. Tal como fue estimado preliminarmente, la potencia de la planta oscila alrededor de $1 \mathrm{MWe}$, estando los valores más bajos alrededor de $750 \mathrm{kWe}$. Este nivel de potencia valida el uso de un modelo de reactor de lecho fijo en equicorriente.

En otro análisis desarrollado, se considera la información de producción de aceite de palma mes a mes reportada por FEDEPALMA para toda Colombia en el año 2015. Se escalan dichos resultados para una planta de procesamiento de aproximadamente 10000 ton/mes. Se analiza la generación de electricidad considerando un $20 \%$ de pérdida de eficiencia cuando el reactor tiene que operar a carga parcial. Para todos los meses, se considera una humedad de biomasa constante de $15 \%$ con ER de 0,33. Debido a falta de información detallada, se asume que la pérdida de eficiencia es lineal entre el punto de máxima y el de mínima disponibilidad del recurso. No se considera almacenamiento del residuo en la empresa debido a los requerimientos de espacio que esto podría conllevar. El cálculo de la demanda se realiza asumiendo que cada ton de RFF procesado requiere aproximadamente $20 \mathrm{kWh}$ de electricidad [3]. No se considera demanda de potencia aparente.

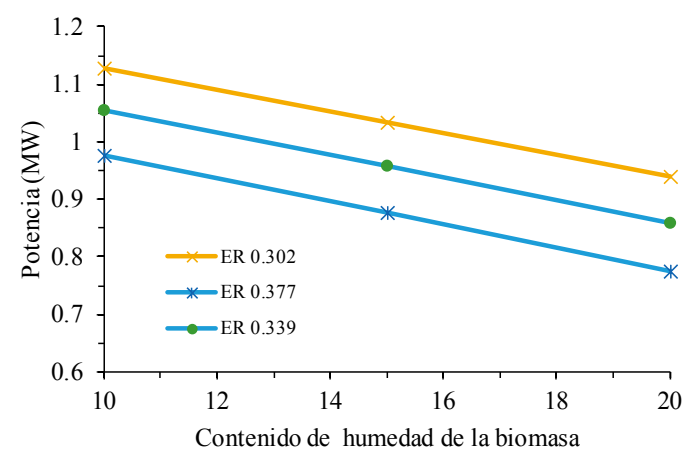

Fig. 6. Potencia eléctrica estimada para el cuesco con diferentes contenidos de humedad y ER. Fuente: autores. 
La Fig. 7 muestra que la totalidad de la demanda puede ser suplida considerando el sistema de gasificación durante los meses de febrero a noviembre. En enero y febrero, la energía generada podría estar por debajo de la demanda debido a la pérdida de eficiencia del sistema al operar a carga parcial, sin embargo, esta situación puede ser evitada disponiendo pequeñas unidades de almacenamiento en las empresas. En los meses de mayor procesamiento, la demanda puede ser suplida con cerca del $80 \%$ del cuesco generado.

La demanda se toma a partir de lo reportado por FEDEPLAMA, mientas que la generación se estima mediante los resultados estimados con el modelo desarrollado.

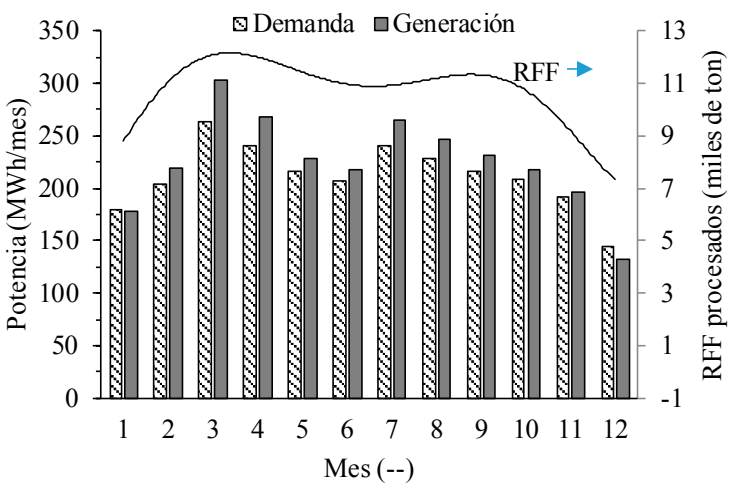

Fig.7. Demanda (primera columna) y generación (segunda columna) de potencia para una planta de procesamiento de 10000 ton RFF mes. Fuente: autores y Fedepalma.

\section{Conclusiones}

En el presente estudio fue analizado el potencial de generación de electricidad basado en los residuos de cuesco provenientes de una extractora de aceite de palma que procese 10000 ton RFF/mes. La alta demanda de vapor de estas industrias se considera que puede ser suplida mediante el aprovechamiento de la fibra, cuya energía primaria es alrededor de 3 veces la requerida para la generación del vapor.

Para el volumen de residuos de cuesco producido, la tecnología que mejor se adapta para la generación de electricidad es gasificación acoplado a motor-generador. Buscando dar confiabilidad a los resultados, se desarrolló un modelo en equilibrio químico mediante el cual se predice de manera adecuada la composición y el volumen de gas producido, parámetros de gran importancia en los análisis de potencial energético. La validación fue realizada comparando los resultados del modelo con los de un estudio teórico-experimental disponible en la literatura. Considerando una relación de equivalencia de 0,33 y $15 \%$ de humedad de la biomasa, se producirían aproximadamente 70 $\mathrm{kg} / \mathrm{h}$ de gas con la siguiente composición: $12,5 \%$ de $\mathrm{H}_{2}, 21,8 \%$ de $\mathrm{CO}, 9,5 \%$ de $\mathrm{CO} 2,56 \%$ de $\mathrm{N}_{2}$ y trazas de $\mathrm{CH}_{4}$, para un PCI de $4,1 \mathrm{MJ} / \mathrm{Nm}_{3}$.
De acuerdo con los resultados del análisis de sensibilidad variando contenido de humedad de la biomasa y relación de equivalencia, la potencia a generar siempre estaría entre 0,8 y 1,1 MWe, siendo el resultado más bajo el correspondiente a alto contenido de humedad y a alta relación de equivalencia, lo cual concuerda con lo reportado en la literatura. El análisis de desempeño de la planta revela que, en 10 de los 12 meses del año, la unidad de generación por gasificación podría suministrar la totalidad de la potencia requerida para la operación de la planta. En los meses de mayor procesamiento, la planta solo requiere el $80 \%$ del cuesco generado.

\section{Agradecimientos}

Agradecemos a la convocatoria 753 de Colciencias (Formación de Capital Humano de Alto Nivel para el Departamento de Norte de Santander), y al departamento Norte de Santander por los recursos destinados para la formación de estudiantes a nivel de Maestría y Doctorado.

\section{REFERENCIAS}

[1] M. La Villetta, M. Costa y N. Massarotti, "Modelling approaches to biomass gasification: A review with emphasis on the stoichiometric method," Renew. Sustain. Energy Rev., vol. 74, no. November 2016, pp. 7188, 2017.https://doi.org/10.1016/j.rser.2017.02.027

[2] M. A. Masmoudi, K. Halouani y M. Sahraoui, "Comprehensive experimental investigation and numerical modeling of the combined partial oxidation-gasification zone in a pilot downdraft air-blown gasifier," Energy Convers. Manag., vol. 144, pp. 34-52, 2017. https://doi. org/10.1016/j.enconman .2017.04.040

[3] E. E. Silva y E. Yáñez, "Potencial de Cogeneración de Energía Eléctrica,” 2007.

[4] J. A. García, M. M. Cárdenas y E. E. Yáñez, "Generación y uso de biomasa en plantas de beneficio de palma de aceite en Colombia Power Generation and Use of Biomass at Palm Oil Mills in Colombia," PALMAS, vol. 31, no. 2, pp. 41-48, 2010 .

[5] J. F. Perez, Y. Lenis, S. Rojas y C. Leon, "Decentralized power generation through biomass gasification: a technical - economic analysis and implications by reduction of CO2 emissions," Rev. la Fac. Ing., pp. 157-169, 2012.

[6] N. A. Samiran, M. N. M. Jaafar, J. H. Ng, S. S. Lam y C. T. Chong, "Progress in biomass gasification technique - With focus on Malaysian palm biomass for syngas production," Renew. Sustain. Energy Rev., vol. 62, pp. 1047-1062, 2016._https://doi.org/10.1016/j. rser.2016.04.049

[7] A. V. Bridgwater, "The technical and economic feasibility of biomass gasification for power generation," Fuel, vol. 74, no. 5, pp. 631-653, 1995. https://doi. org/10.1016/0016-2361(95)00001-L

[8] T. Gröbl, H. Walter y M. Haider, "Biomass steam gasification for production of SNG - Process design and sensitivity analysis," Appl. Energy, vol. 97, pp. 451-461, 2012.https://doi.org/10.1016/j.apenergy.2012.01.038

[9] F. Paviet, F. Chazarenc y M. Tazerout, "Thermo Chemical Equilibrium Modelling of a Biomass Gasifying Process Using ASPEN PLUS," Int. J. Chem. React. Eng., vol. 7, p. A40, 2009.„https://doi.org/10.2202/15426580.2089 
[10] P. Kuo, W. Wu y W. Chen, "Gasification performances of raw and torrefied biomass in a downdraft fixed bed gasifier using thermodynamic analysis," Fuel, vol. 117, pp. 1231-1241, 2014. https://doi.org/10.1016/j. fuel.2013.07.125

[11] C. He, X. Feng, K. H. Chu, A. Li y Y. Liu, "Industrialscale fixed-bed coal gasification: Modeling, simulation and thermodynamic analysis," Chinese J. Chem. Eng., vol. 22, no. 5, pp. 522-530, 2014. https://doi. org/10.1016/S1004-9541(14)60066-5

[12] S. Begum, M. G. Rasul y D. Akbar, "A numerical investigation of municipal solid waste gasification using aspen plus," Procedia Eng., vol. 90, pp. 710-717, 2014. https://doi.org/10.1016/j.proeng.2014.11.800

[13] A. Gagliano, F. Nocera, M. Bruno y G. Cardillo, "Development of an Equilibrium-based Model of Gasification of Biomass by Aspen Plus," Energy Procedia, vol 111, pp. 1010-1019, 2017. https://doi.org/10.1016/j.egypro.2017.03.264

[14] N. Deng et al., "Simulation analysis of municipal solid waste pyrolysis and gasification based on Aspen plus," Bioresour. Technol., vol. 235, pp. 371-379, 2017.

[15] A. Alembath, "Aspen simulation of oil shale and biomass process in partial fulfillment of the requirements for the degree," 2016.

[16] T. H. Jayah, L. Aye, R. J. Fuller y D. F. Stewart, "Computer simulation of a downdraft wood gasifier for tea drying," Biomass and Bioenergy, vol. 25, no. 4, pp. 459-469, 2003. https://doi.org/10.1016/S09619534(03)00037-0

[17] S. Jarungthammachote y A. Dutta, "Thermodynamic equilibrium model and second law analysis of a downdraft waste gasifier," Energy, vol. 32, no. 9, pp. 1660-1669, 2007. https://doi.org/10.1016/j.energy.2007.01.010

[18] C. R. Altafini, P. R. Wander y R. M. Barreto, "Prediction of the working parameters of a wood waste gasifier through an equilibrium model," Energy Convers. Ma nag., vol. 44, no. 17, pp. 2763-2777, 2003. https://doi. org/10.1016/S0196-8904(03)00025-6

[19] I. Briceño, J. Valencia y M. Posso, "Potencial de generación de energía de la agroindustria de la palma de aceite en Colombia," Palmas, vol. 36, pp. 43-53, 2015.

[20] Fedepalma, "Desempeño del sector palmero colombia no," 2016.

[21] N. Ramzan, A. Ashraf, S. Naveed y A. Malik, "Simulation of hybrid biomass gasification using Aspen plus : A comparative performance analysis for food, municipal solid and poultry waste," Biomass and Bioenergy, vol. 35, no. 9, pp. 3962-3969, 2011. https://doi. org/10.1016/j.biombioe.2011.06.005

[22] D. Che, S. Li, W. Yang, J. Jia y N. Zheng, "Application of Numerical Simulation on Biomass Gasification," Energy Procedia, vol. 17, pp. 49-54, 2012. https://doi. org/10.1016/j.egypro.2012.02.061

[23] R. Nayak y R. K. Mewada, "Simulation of Coal Gasifi cation Process using ASPEN PLUS," pp. 8-10, 2011.

[24] R. F. E. Machorro, "Estudio de la producción de hidrógeno a partir de los residuos sólidos de la ciudad de méxico mediante la tecnología de gasificación,” 2016.

[25] M. Fernández-López, J. Pedroche, J. L. Valverde y L. Sánchez-Silva, "Simulation of the gasification of animal wastes in a dual gasifier using Aspen Plus," Energy Convers. Manag., vol. 140, pp. 211-217, 2017. https:// doi.org/10.1016/j.enconman.2017.03.008

[26] A. A. P. Susastriawan y H. Saptoadi, "Small-scale downdraft gasi fi ers for biomass gasi fi cation: A review," vol. 76, no. March, pp. 989-1003, 2017. https:// doi.org/10.1016/j.rser.2017.03.112
Daniel Andrés Quintero Coronel. Ingeniero mecánico egresado de la Universidad Francisco de Paula Santander. Actualmente se encuentra desarrollando una maestría en Ingeniería mecánica en la Universidad del Norte de la ciudad de Barranquilla. Pertenece al grupo de investigación UREMA (Uso Racional y Preservación de Energía y Medio Ambiente). https:// orcid.org/0000-0003-2822-5984

Yuhan Lenis Rodas. Ingeniero mecánico, Universidad de Antioquia, 2009 (Medellín, Colombia). Magíster en ingeniería de la misma universidad en el año 2013. Actualmente es estudiante de doctorado en Ingeniería mecánica de la Universidad del Norte. Entre 2007 y 2017 participó en investigaciones en el área de ciencias térmicas, analizando motores de combustión interna y procesos de gasificación de biomasa. Se encuentra vinculado a los grupos de investigación GIMEL (Universidad de Antioquia, 2007-2014), Termomec (Universidad Cooperativa de Colombia, 2013-2016), GREEN (Universidade de São Paulo, 2017-Actual) y UREMA (Universidad del Norte, 2014-actualmente). Al presente, sus líneas de profundización incluyen: energía, gasificación de biomasa, biocombustibles, simulación de procesos térmicos, energía solar concentrada y control automático de procesos. https://orcid.org/0000-00032468-7874

Lesme Corredor Martínez. Ingeniero mecánico, Universidad del Norte, 1989 (Barranquilla, Colombia). Doctor en Ingeniería mecánica, Universidad Politécnica de Madrid (España), 1999. En la actualidad se desempeña como profesor de tiempo completo del Departamento de Ingeniería Mecánica de la Universidad del Norte (Barranquilla-Colombia). Su ejercicio profesional se ha centrado en dos frentes: 1) industrial, en el diseño e implementación de sistema de cogeneración y/o trigeneración orientados a la optimización energética de los procesos y a la reducción de gases efecto invernadero de los mismos, tales sistemas alimentados, ya sea con combustibles fósiles o renovables; y 2) sector transporte, estudios teórico-experimentales dirigidos a la sustitución de combustibles líquidos y a la incorporación de tecnologías bajas en carbono en el sector transporte. Ha dirigido y participado en varios proyectos universidad-empresa en todo lo relacionado con el uso eficiente de la energía. En el campo de las fuentes renovables, ha desarrollado varias investigaciones en la producción y utilización en motores diesel de biodiesel a partir de aceite de palma y bioetanol, al igual que el diseño e implementación de sistemas de trigeneración alimentados con biomasa residual de palma africana. Fue consejero del Programa de Energía y Minería de Colciencias (2003-2006) y es miembro del Consejo Mundial de Energía. https:// orcid.org/0000-0002-3037-739X 